\title{
.INFINITE CONVOLUTIONS ON LOCALLY COMPACT ABELIAN GROUPS AND ADDITIVE FUNCTIONS $\left({ }^{1}\right)$
}

BY

\author{
PHILIP HARTMAN
}

\begin{abstract}
Let $\mu_{1}, \mu_{2}, \ldots$ be regular probability measures on a locally compact Abelian group $G$ such that $\mu=\mu_{1} * \mu_{2} * \cdots=\lim \mu_{1} * \cdots * \mu_{n}$ exists (and is a probability measure). For arbitrary $G$, we derive analogues of the Lévy theorem on the existence of an atom for $\mu$ and of the "pure theorems" of Jessen, Wintner and van Kampen (dealing with discrete $\mu_{1}, \mu_{2}, \ldots$ ) in the case $G=R^{d}$. These results are applied to the asymptotic distribution $\mu$ of an additive function $f: Z_{+} \rightarrow G$ after generalizing the Erdös-Wintner result $\left(G=R^{1}\right)$ which implies that $\mu$ is an infinite convolution of discrete probability measures.
\end{abstract}

1. Introduction. Let $\mu_{1}, \mu_{2}, \ldots$ be regular probability measures on $R^{d}$ such that

$$
\mu=\lim _{n \rightarrow \infty} \mu_{1} * \cdots * \mu_{n}=\mu_{1} * \mu_{2} * \cdots
$$

is convergent. A result of P. Lévy [11, Theorem XIII, p. 150] states that $\mu$ is not continuous (i.e., has an atom) if and only if

$$
\prod_{n=1}^{\infty} d_{n} \neq 0, \quad \text { where } d_{n}=\max _{t} \mu_{n}(\{t\})
$$

is the largest "jump" of $\mu_{n}$. Also, a theorem of Jessen and Wintner [9, Theorem 35, p. 86] states that if $\mu_{n}$ is purely discontinuous (= discrete), then $\mu$ is purely discontinuous or absolutely continuous or (continuous) singular and, more generally (van Kampen [10, pp. 443-444]), $\mu$ is pure; cf. $\$ 2$ below. In $\S \S 2$ and 3, we discuss generalizations of these results when $R^{d}$ is replaced by a locally compact Abelian group $G$. Our methods follow van Kampen's treatment [10] of infinite convolutions on $R$; cf. also Jessen and Wintner [9], and Wintner [16] , [17].

For example, our results imply that in the case when $G$ is the circle group

Received by the editors September 13, 1974.

AMS (MOS) subject classifications (1970). Primary 60B15; Secondary 10K99.

Key words and phrases. Locally compact Abelian groups, regular probability measures, infinite convolutions, absolutely continuous, purely discontinuous (= discrete), singular measures, additive functions, asymptotic distributions.

(1) Research supported in part by NSF Grant GP-30483. 
$T=R / Z$, where every closed subgroup $H(\neq G)$ is finite, the analogue of the Jessen-Wintner (and van Kampen) result is valid, but the analogue of the Lévy theorem has the following form: $\mu$ is not continuous if and only if there exists an integer $\kappa>0$ such that

$$
\prod_{n=1}^{\infty} d_{\kappa n} \neq 0, \text { where } d_{\kappa n}=\max _{\theta} \sum_{j=0}^{\kappa-1} \mu_{n}(\{\theta+j / \kappa\}) .
$$

The motivation for dealing with (1.1) when $\mu, \mu_{1}, \mu_{2}, \ldots$ are probability measures on a group arises, for example, from the consideration of the asymptotic distribution functions of real-valued additive functions mod 1 or, more generally, of additive functions $f: Z_{+} \rightarrow G$, where $Z_{+}=\{1,2, \ldots\}$. A result of Erdös and Wintner [6, p. 720] states that if $G=R$, then $f$ has an asymptotic distribution $\mu$ if and only if (1.1) converges, where $\mu_{n}=\sigma_{p}$ is purely discontinuous and has the Fourier-Stieltjes transform

$$
\hat{\sigma}_{p}(u)=\left(1-p^{-1}\right)\left[1+\sum_{j=1}^{\infty} p^{-j} \exp \operatorname{iuf}\left(p^{j}\right)\right],
$$

and $p=p_{n}$ is the $n$th prime. This is generalized in $\S 4$ to the case of arbitrary locally compact Abelian groups $G$. In particular, it follows in the case $G=T$ (as in the Erdös-Wintner case $G=R$ ) that when $\mu$ exists, it is pure (hence absolutely continuous or purely discontinuous or (continuous) singular). $\$ 4$ depends heavily on results of Halasz [7], and their applications by Delange [3].

This article was suggested by the paper of Elliott [5] dealing with the question of the continuity of the asymptotic distribution of a real additive function mod 1 (using results of Halasz and Delange, but not involving convolutions).

2. Cauchy-convergent convolutions on groups. Let $G$ be a (Hausdorff) locally compact Abelian group (written additively) and $\Gamma$ the dual group of continuous characters. We write $(g, \gamma)$ for the pairing of $G$ and $\Gamma, g \in G$ and $\gamma \in \Gamma$. Let $P(G)$ be the set of regular probability measures $\mu$ on $G$. The Fourier-Stieltjes transform of $\mu$ is

$$
\hat{\mu}(\gamma)=\int_{G}(g, \gamma) d \mu \quad \text { for } \gamma \in \Gamma .
$$

For $\mu, \nu \in P(G)$, we have $(\mu * \nu)^{\wedge}(\gamma)=\hat{\mu}(\gamma) \hat{\nu}(\gamma)$; cf. [15, pp. 13-15].

The standard topology on $P(G)$ is equivalent to the following: for any net $\left\{\mu_{n}\right\}$ in $P(G)$ and $\mu \in P(G), \mu_{n} \rightarrow \mu$ in $P(G)$ is equivalent to

$$
\int_{G} f(g) d \mu_{n} \rightarrow \int_{G} f(g) d \mu \quad \text { for all } f \in C_{0}^{0}(G),
$$

where $C_{0}^{0}(G)$ is the set of complex-valued continuous functions on $G$ with compact support $[1$, p. 82]. Furthermore, $(2.1)$ can be replaced by any of the fol- 
lowing three equivalent conditions on Fourier-Stieltjes transforms, where $\mu_{n}, \mu \in$ $P(G)$ :

(i) $\hat{\mu}_{n}(\gamma) \rightarrow \hat{\mu}(\gamma)$ uniformly on compacts of $\Gamma$;

(ii) $\hat{\mu}_{n}(\gamma) \rightarrow \hat{\mu}(\gamma)$ for all $\gamma \in \Gamma$;

(iii) $\int_{\Gamma} f(\gamma) \hat{\mu}_{n}(\gamma) d \gamma \rightarrow \int_{\Gamma} f(\gamma) \hat{\mu}(\gamma) d \gamma$ for all $f \in L^{1}(\Gamma)$,

and $L^{1}(\Gamma)$ refers to a Haar measure on $\Gamma$; cf. [1, p. 89] (where $G=\hat{\Gamma}$ and $\Gamma=\hat{G}$ are interchanged).

Also, if $\left\{\mu_{n}\right\}$ is a net in $P(G)$, then $\lim \mu_{n}$ exists in $P(G)$ if and only if $\lim \hat{\mu}_{n}(\gamma)$ exists for all $\gamma \in \Gamma$ and is continuous at $\gamma=0$.

In fact, the limit function is then continuous on $\Gamma$ by the analogue of the Increments Inequality (cf. Loève $[12$, p. 195]),

$$
|\hat{\mu}(\gamma)-\hat{\mu}(\gamma+\delta)|^{2} \leqslant 2[1-\operatorname{Re} \hat{\mu}(\delta)] \quad \text { for } \gamma, \delta \in \Gamma, \mu \in P(G),
$$

which holds for $\hat{\mu}=\hat{\mu}_{n}$ and hence for $\hat{\mu}=\lim \hat{\mu}_{n}$. And $\lim \hat{\mu}_{n}$, being continuous and positive definite with the value 1 at $\gamma=0$, is the Fourier-Stieltjes transform $\hat{\mu}$ of some $\mu \in P(G)$ (Bochner, cf. [15, p. 19]) and satisfies (ii) above.

We write $\{g\}$ for the subset of $G$ consisting of the point $g$, so that $g$ is an atom if $\mu(\{g\})>0$. We write $\omega_{1}=\omega_{1 G} \in P(G)$ for the unit measure (i.e., $\left.\omega_{1}(\{0\})=1\right)$ and $\omega_{0 G}$ for [normalized] Haar measure on $G$ [if $G$ is compact], so that $\omega_{0 G} \in P(G)$ if $G$ is compact. Also

$$
\hat{\omega}_{1}(\gamma)=1 \text { and if } G \text { is compact, } \hat{\omega}_{0 G}(\gamma)=0 \text { for } \gamma \neq 0 \text {. }
$$

Proposition 2.1. Let $\mu, \nu \in P(G)$. The set of atoms [or support] of $\mu * \nu$ may be obtained by adding arbitrary elements of the sets of atoms [or supports] of $\mu$ and $\nu$ [and forming the closure]. Also $(\mu * \nu)(\{g\})=\Sigma \mu(\{x\}) \nu(\{y\})$ for $x+y=g$. If $\mu_{n} \rightarrow \mu$ in $P(G)$ as $n \rightarrow \infty$ and $\Sigma(\mu)$ denotes the support of $\mu$, then $\Sigma(\mu) \subset \lim \Sigma\left(\mu_{n}\right)$ as $n \rightarrow \infty$.

By $\lim \Sigma\left(\mu_{n}\right)$ as $n \rightarrow \infty$, we mean the set of points $g \in G$ with the property that, for every neighborhood $U$ of $g, U \cap \Sigma\left(\mu_{n}\right) \neq \varnothing$ for large $n$. The next proposition follows by considering Fourier-Stieltjes transforms.

Proposition 2.2. If $\mu_{1}, \mu_{2}, \ldots \in P(G)$ satisfy

$$
\mu_{n} * \cdots * \mu_{N} \rightarrow \omega_{1} \text { as } N \geqslant n \rightarrow \infty,
$$

then

$$
\lim _{n \rightarrow \infty} \mu_{1} * \cdots * \mu_{n}=\mu \text { exists in } P(G) .
$$

In contrast to the case of convolutions on $R^{d},(2.6)$ does not imply (2.5). This is clear if $G$ is compact and $\omega_{0 G}$ is its normalized measure for, by (2.4), $\omega_{0 G} * \mu_{1} * \cdots * \mu_{n}=\omega_{0 G} \rightarrow \omega_{0 G}$ as $n \rightarrow \infty$ for arbitrary $\mu_{1}, \mu_{2}, \ldots$. 
Definition. When (2.6) holds, we say that the infinite convolution $\mu=$ $\mu_{1} * \mu_{2} * \cdots$ is convergent. If, in addition, (2.5) holds, we say that it is Cauchyconvergent.

Proposition 2.3. If $\mu=\mu_{1} * \mu_{2} * \cdots$ is Cauchy-convergent, then $\Sigma(\mu)=$ $\lim \Sigma\left(\mu_{1} * \cdots * \mu_{n}\right)$ as $n \rightarrow \infty$.

The proof can be obtained by a modification of that of Wintner [16, pp. 60-61] for $R$. We consider analogues of Lévy's theorem for Cauchy-convergent convolutions.

THEOREM 2.1. (i) If $\mu=\mu_{1} * \mu_{2} * \cdots$ is convergent and

$$
\prod_{n=1}^{\infty} d_{0 n} \neq 0, \text { where } d_{0 n}=\max _{g} \mu_{n}(\{g\})
$$

then $\mu$ is not continuous (i.e., $\mu$ has at least one atom). (ii) Conversely, if $\mu=$ $\mu_{1} * \mu_{2} * \cdots$ is Cauchy-convergent and $\mu$ is not continuous, then (2.7) holds.

The following is similar to the proof of P. Lévy [11, pp. 150-152] as simplified by Jessen; cf. van Kampen [10, pp. 445-446] or Wintner [16, pp. 16 $-18]$.

Proof. On (i). Let $g_{n} \in G$ satisfy $\Pi \mu_{n}\left(\left\{g_{n}\right\}\right)=d>0$, e.g., let $d_{0 n}=$ $\mu_{n}\left(\left\{g_{n}\right\}\right)$. Let $\lambda_{n}=\mu_{1} * \cdots * \mu_{n}$, so that

$$
\lambda_{n}\left(\left\{h_{n}\right\}\right) \geqslant \prod_{k=1}^{n} \mu_{k}\left(\left\{g_{k}\right\}\right) \geqslant d,
$$

where $h_{n}=g_{1}+\cdots+g_{n}$. There exists a compact $K \subset G$ which contains all but a finite number of $h_{1}, h_{2} \cdots$. For otherwise, if $K$ is any compact, then $\lambda_{n}(K)$ $\leqslant 1-d$ for infinitely many $n$. Thus $\mu(K) \leqslant 1-d$ for any compact $K$; so that, since $K$ is arbitrary, we obtain the contradiction $\mu(G) \leqslant 1-d<1$. Thus $h_{1}, h_{2}$, $\cdots$ has a cluster point $g$. If $U$ is any neighborhood of $0 \in G$, then $\lambda_{n}(g+U) \geqslant$ $d$ for infinitely many $n$, and so $\mu(g+U+U) \geqslant d$. Thus $\mu(\{g\}) \geqslant d>0$.

On (ii). Let $\mu\left(\left\{g_{0}\right\}\right)=d>0$. Following the arguments of $[10,(18), p$. 445] we can obtain:

(a) Let $0<d \leqslant 1,0<6 \epsilon<d$ and $U$ be a symmetric compact neighborhood of $0 \in G$. Let $\lambda, \mu, \nu \in P(G)$ with the properties

$$
\begin{aligned}
& \mu=\lambda * \nu \text { and } \mu\left(\left\{g_{0}\right\}\right)=d \text { for some } g_{0} \in G, \\
& \mu\left(g_{0}+U+U\right)<d+\epsilon \text { and } \nu(U)>1-\epsilon .
\end{aligned}
$$

Then there exist $g, h \in G$ such that $g_{0}=g+h, h \in U$,

$$
d-\epsilon<\lambda(\{g\})<(d+\epsilon) /(1-\epsilon) \text { and } \nu(\{h\})>1-6 \epsilon / d .
$$


For $n=1,2, \ldots$, put $\lambda_{n}=\mu_{1} * \mu_{2} * \cdots * \mu_{n}$. Also $\nu_{n}=\mu_{n+1} * \mu_{n+2}$ $* \cdots$ is defined and Cauchy-convergent, and $\mu=\lambda_{n} * \nu_{n}$ and $\nu_{n} \rightarrow \omega_{1}$ as $n \rightarrow$ $\infty$. We now verify the following assertion; cf. [10, (19), p. 446]. (Curiously, no assumption of metrizability of $G$ is required.)

(b) There exist $g_{1}, g_{2}, \ldots$ and $h_{1}, h_{2}, \ldots$ in $G$ such that $g_{0}=g_{n}+h_{n}$ and $g_{n} \rightarrow g_{0}, h_{n} \rightarrow 0$,

$$
\lambda_{n}(\{g\}) \rightarrow d \text { and } \nu_{n}\left(\left\{h_{n}\right\}\right) \rightarrow 1 \text { as } n \rightarrow \infty .
$$

Let $D \geqslant 2$ be an integer such that $D d>6$, and for $m=D, D+1, \ldots$, choose symmetric compact neighborhoods $U_{D}, U_{D+1}, \ldots$ of $0 \in G$ such that $U_{D} \subset U_{D+1} \subset \cdots$ and $\mu\left(g_{0}+U_{m}+U_{m}\right)<d+1 / m$, and choose $N_{D}<N_{D+1}$ $<\cdots$ so that $\nu_{n}\left(U_{m}\right)>1-1 / m \geqslant 1 / 2$ for $n \geqslant N_{m}, m \geqslant D$. Then, by (a), there exist $g_{n m}, h_{n m} \in G$ for $n \geqslant N_{m}$ satisfying $g_{0}=g_{n m}+h_{n m}, h_{n m} \in U_{m}$,

$$
d-1 / m<\lambda_{n}\left(\left\{g_{n m}\right\}\right)<(d+1 / m) /(1-1 / m) \text { and } \nu_{n}\left(\left\{h_{n m}\right\}\right)>1-6 / d m \text {. }
$$

Thus, $\lambda_{n}\left(\left\{g_{n m}\right\}\right) \rightarrow d$ and $\nu_{n}\left(\left\{h_{n m}\right\}\right) \rightarrow 1$ for $n \geqslant N_{m}, m \rightarrow \infty$. For $n \geqslant N_{D}$, let $m=m(n)$ satisfy $N_{m} \leqslant n<N_{m+1}$. Put $g_{n}=g_{n, m(n)}$ and $h_{n}=h_{n, m(n)}$, so that (2.8) holds. Suppose that the sequence $h_{D}, h_{D+1}, \ldots \in U_{D}$ has a cluster point $g \neq 0$. If $U$ is a compact neighborhood of $g$, then $\nu_{n}(U) \geqslant \nu_{n}\left(\left\{h_{n}\right\}\right) \geqslant 1-$ $6 / d m(n)$ for infinitely many $n$. But if $0 \notin U$, then $\nu_{n}(U) \rightarrow \omega_{1}(U)=0$ as $n \rightarrow$ $\infty$. This contradiction gives $h_{n} \rightarrow 0$ as $n \rightarrow \infty$, and proves (b).

Assertion (ii) can be proved by the use of (b) in the same way that Lévy's case of $G=R$ is proved in [10] with the use of the equivalent [10, (19), p. 446]. We omit details.

Let $\mu \in P(G)$. If $(X, \Omega, \sigma)$ is a probability measure space, a map $\phi: X \rightarrow G$ is called measurable if $\phi^{-1}(A) \subset X$ is measurable for every Borel set $A \subset G$. If, also $\phi:(X, \sigma) \rightarrow(G, \mu)$ is a measure preserving map (i.e., $\sigma\left(\phi^{-1}(A)\right)=\mu(A)$ for all Borel sets $A \subset G$ ), then $\mu$ is called the distribution of $\phi$. It is clear that if $\mu$ is purely discontinuous, then there exist probability spaces $(X, \Omega, \sigma)$ and maps $\phi: X \rightarrow G$ such that $\phi$ has $\mu$ as its distribution. (More generally, this is the case if $(G, \mu)$ is a Lebesgue measure space; cf. [14].)

Let $(X, \Omega, \sigma)$ be a probability space and $s_{1}(x), s_{2}(x), \ldots$ a sequence of measurable maps $s_{n}: X \rightarrow G$. We say that $s_{1}(x), s_{2}(x), \ldots$ is Cauchy in measure if, for every neighborhood $U$ of $0 \in G$,

$$
\sigma\left\{x \in X: s_{N}(x)-s_{n}(x) \notin U\right\} \rightarrow 0 \text { for } N>n \rightarrow \infty .
$$

If this is the case and, in addition, $G$ is metrizable, then standard proofs for $G=R$ show that there exists a measurable map $s: X \rightarrow G$ such that $s_{n}(x) \rightarrow$ $s(x)$ in measure as $n \rightarrow \infty$, i.e., 


$$
\sigma\left\{x \in X: s_{n}(x)-s(x) \notin U\right\} \rightarrow 0 \text { as } n \rightarrow \infty .
$$

Also there exists a subsequence $s_{n(1)}(x), s_{n(2)}(x), \ldots$ satisfying

$$
s_{n(j)}(x) \rightarrow s(x) \text { a.e. on }(X, \Omega, \sigma) \text { as } j \rightarrow \infty \text {. }
$$

We adopt the conventions of [10], omitting details here. Let $X=X_{1} \times$ $X_{2} \times \cdots$ be an infinite product measure space carrying a product measure $\sigma=$ $\Pi \sigma_{n}$, each $X_{n}$ is a probability measure space with measure $\sigma_{n}$. A point $x \in X$ is a sequence $x=\left(x_{1}, x_{2}, \ldots\right)$ with $x_{n} \in X_{n}$ and, for example, a function $\phi_{n}\left(x_{n}\right)$ on $X_{n}$ is also considered a function of $x \in X$ independent of $x_{k}, k \neq n$. Let $\mu_{1}, \mu_{2}, \ldots \in P(G)$ and let $\phi_{n}: X_{n} \rightarrow G$ be a function having $\mu_{n}$ as its distribution. Then

$$
s_{n}(x)=\phi_{1}\left(x_{1}\right)+\cdots+\phi_{n}\left(x_{n}\right),
$$

considered as a function on $X$, has $\mu_{1} * \cdots * \mu_{n}$ as its distribution.

PROPOSITION 2.4. Let $\mu_{1}, \mu_{2}, \ldots \in P(G)$ and $X=X_{1} \times X_{2} \times \cdots$, $\phi_{1}\left(x_{1}\right), \phi_{2}\left(x_{2}\right), \ldots$ as above. (i) Then $s_{1}(x), s_{2}(x), \ldots$ is Cauchy in measure on $X$ if and only if $\mu=\mu_{1} * \mu_{2} * \cdots$ is Cauchy-convergent. (ii) If this holds and, in addition, $G$ is metrizable, then $s_{1}(x), s_{2}(x), \ldots$ has a limit $s(x)$ in measure on $X$ and $\mu$ is the distribution of $s(x)$.

Part (i) is clear, for the distribution of $s_{N}(x)-s_{n-1}(x)=\phi_{n}\left(x_{n}\right)+\cdots+$ $\phi_{N}\left(x_{N}\right)$ is $\mu_{n} * \cdots * \mu_{N}$ for $N \geqslant n$. Part (ii) follows from the remarks concerning (2.10).

Following van Kampen [10], we define a pure probability measure $\mu \in P(G)$. Let $\mathscr{Q}$ be a class of Borel sets on $G$ which is closed under countable unions and with the property that if $A \in \mathfrak{A}$, then every translate $A+g \in \mathfrak{A}$. (Such classes are, for instance, the class of enumerable sets or the class of null sets with respect to Haar measure $\omega_{0 G}$.) $\mu \in P(G)$ is said to be pure if it has the following property with reference to every class $\mathfrak{Q}:$ If $\mu(A)>0$ for some $A \in \mathfrak{U}$, then there exists an $A_{0} \in \mathscr{U}$ such that $\mu\left(A_{0}\right)=1$.

A probability measure $\mu \in P(G)$ is called continuous if it has no atoms (i.e., $\mu(\{g\})=0$ for all $g \in G)$, purely discontinuous if $\mu(A)=1$ for some enumerable set $A$, absolutely continuous (with respect to Haar measure $\omega_{0 G}$ ) if $\mu(A)=0$ whenever $\omega_{0 G}(A)=0$ and, finally, (continuous) singular if it is continuous and if $\mu\left(A_{0}\right)=1$ for some set $A_{0}$ with $\omega_{0 G}\left(A_{0}\right)=0$. [Note that $\mu \neq 0$ is absolutely continuous and purely discontinuous if $G$ is countable.]

TheOREM 2.2 (PURE THEOREM). Let $\mu_{n} \in P(G)$ be purely discontinuous and $\mu=\mu_{1} * \mu_{2} * \cdots$ Cauchy-convergent. Then $\mu$ is pure (hence absolutely continuous or purely discontinuous or (continuous) singular). 
If $G$ is metrizable, this result follows from Proposition 2.4(ii) and the 0-or-1 principle; cf. the proof of [9, Theorem 35, p. 86] or [10, Theorem VIII, p. 444]. We shall modify these arguments, using Proposition 2.4(i), avoiding a "limit a.e." or "limit in measure". (Roughly speaking, we consider an arbitrary, but fixed, symmetric neighborhood $V$ of $0 \in G$, a sequence $V_{0}, V_{1}, \ldots$ of such neighborhoods with $V=V_{0}$ and $V_{k+1}+V_{k+1} \subset V_{k}$ and the pseudo-metric induced on $G$ by the neighborhood "base" $V_{0}, V_{1}, \ldots$ of $0 \in G$.)

Proof. We give the proof in several steps. We write $A(2)=A+A, A(3)$ $=A+A+A$, etc. If $Y \subset X$, we write $Y^{c}$ for the complement of $Y$ in $X$.

Let $X=X_{1} \times X_{2} \times \cdots$ and $\phi_{1}\left(x_{1}\right), \phi_{2}\left(x_{2}\right), \ldots$ be as in Proposition 2.4(i). Since $\phi_{n}$ is purely discontinuous, there is no difficulty about the existence of $X_{n}$ and $\phi_{n}$. It can also be supposed that the range of $\phi_{n}\left(x_{n}\right)$ in $G$ is countable. Let $M$ be a countable subset of $G$ containing the ranges of $s_{n}$ and $s_{n}-s_{m}$ for $n, m=$ $1,2, \ldots$.

(a) Let $V$ be a symmetric neighborhood of $0 \in G$. Then there exists a sequence of integers $0<n(1)<n(2)<\cdots$, depending on $V$, with the following property: if $\epsilon>0$, then there exist an integer $N_{\epsilon}=N_{\epsilon V}$ and a measurable set $X_{\epsilon}=X_{\epsilon V} \subset X$ such that $\sigma\left(X_{\epsilon}\right)>1-\epsilon$ and

$$
s_{n(j)}(x)-s_{n(k)}(x) \in V \text { for } x \in X_{\epsilon} \text { and } n(j), n(k) \geqslant N_{\epsilon} \text {. }
$$

In order to see this, let $V_{0}, V_{1}, \ldots$ be a sequence of symmetric neighborhoods of $0 \in G$ such that $V=V_{0}$ and $V_{k+1}(2) \subset V_{k}$ for $k=0,1, \ldots$, so that $\left(V_{1}+\cdots+V_{k}\right)+V_{k} \subset V$ for $k=1,2, \ldots$. Choose $0<n(1)<n(2)<\cdots$, so that

$$
\sigma\left(\left\{x \in X: s_{n}(x)-s_{m}(x) \notin V_{k}\right\}\right)<1 / 2^{k} \quad \text { for } n, m \geqslant n(k) .
$$

If $K$ is so large that $2 / 2^{K}<\epsilon$ and if

$$
X_{\epsilon}=\left[\bigcap_{k=K}^{\infty}\left\{x \in X: s_{n(k+1)}(x)-s_{n(k)}(x) \notin V_{k}\right\}\right]^{c},
$$

then $\sigma\left(X_{\epsilon}^{c}\right)<1 / 2^{K}+1 / 2^{K+1}+\cdots=2 / 2^{K}<\epsilon$. Also, if $N_{\epsilon}=n(K)$, then $x \in X_{\epsilon}$ and $n(j)>n(k) \geqslant N_{\epsilon}$ imply that

$$
\pm\left(s_{n(j)}(x)-s_{n(k)}(x)\right) \in V_{k}+V_{k+1}+\cdots+V_{j-1} \subset V .
$$

This gives (a).

(b) In the remainder of the proof, except for the last two sentences, $V$ is fixed. We can therefore suppose that $n(1), n(2), \ldots$ is the full sequence 1,2 , $\ldots$, for otherwise we replace $\mu_{1} * \mu_{2} * \cdots$ by $\left[\mu_{1} * \cdots * \mu_{n(1)}\right] *\left[\mu_{n(1)+1}\right.$ $\left.* \cdots * \mu_{n(2)}\right] * \cdots, X_{1} \times X_{2} \times \cdots$ by $\left[X_{1} \times \cdots \times X_{n(1)}\right] \times\left[X_{n(1)+1} \times\right.$ $\left.\cdots \times X_{n(2)}\right] \times \cdots$, and $\phi_{1}, \phi_{2}, \ldots$ by $s_{n(1)}, s_{n(2)}-s_{n(1)}, \ldots$. 
For a subset $A$ of $G$, introduce the following subsets of $X$ :

$D_{n}(A, V)=\left\{x \in X: s_{n}(x) \in A+V\right\}=s_{n}^{-1}(A+V)$,

$D(A, V)=\left\{x \in X: s_{n}(x) \in A+V\right.$ for large $\left.n\right\}=\bigcup_{k=1}^{\infty} \bigcap_{n=k}^{\infty} D_{n}(A, V)$.

(c) For $j>0, D(A, V(j)) \cap X_{\epsilon} \subset D_{n}(A, V(j+1))$, hence $D(A, V(j)) \subset$ $D_{n}(A, V(j+1)) \cup X_{\epsilon}^{c}$, for $n \geqslant N_{\epsilon}$. For if $x \in D(A, V(j))$, then $s_{m}(x) \in A+$ $V(j)$ for large $m$, and if $x \in X_{\epsilon}$, then $s_{n}(x)-s_{m}(x) \in V$ for $n, m \geqslant N_{\epsilon}$. Thus, $x \in D(A, V(j)) \cap X_{\epsilon}$ implies that $s_{n}(x) \in s_{m}(x)+V \subset A+V(j)+V=A+$ $V(j+1)$ for $n \geqslant N_{\epsilon}$ and large $m$; i.e., $x \in D_{n}(A, V(j+1))$.

(d) For $j>0, D_{n}(A, V(j)) \cap X_{\epsilon} \subset D(A, V(j+1))$, hence $D(A, V(j)) \subset$ $D(A, V(j+1)) \cap X_{\epsilon}^{c}$, for $n \geqslant N_{\epsilon}$. For if $x \in D_{n}(A, V(j))$, then $s_{n}(x) \in A+V(j)$. Thus, $x \in D_{n}(A, V(j)) \cap X_{\epsilon}$ implies that $s_{m}(x) \in s_{n}(x)+V \subset A+V(j+1)$ for $m \geqslant n \geqslant N_{\epsilon}$; i.e., $x \in D(A, V(j+1)$ ). This gives (d) which together with (a), (b), and (c) have the following consequences.

(e) Let $A \subset G$ be a Borel set and $\lambda_{n}=\mu_{1} * \cdots * \mu_{n}$. Then $\sigma(D(A, V(2)))$ $\leqslant \sigma\left(D_{n}(A, V(3))\right)+\epsilon=\lambda_{n}(A+V(3))+\epsilon$ and $\lambda_{n}(A+V)=\sigma\left(D_{n}(A, V)\right) \leqslant$ $\sigma(D(A, V(2)))+\epsilon$ for $n \geqslant N_{\epsilon}$.

(f) COMPLETION OF THE PROOF. Let $\mathfrak{A}$ be an admissible class of Borel subsets of $G$ and suppose that $\mu(A)>0$ for some $A \in \mathfrak{U}$. Then $\lambda_{n}(A+V) \geqslant$ $\mu(A) / 2>0$ for large $n$. Let $A_{0}=A+M=\bigcup(A+g)$ for $g \in M$, so that $A_{0} \in$ Q⿱ $)$ since $M$ is countable. If $0<\epsilon<\mu(A) / 2$, then $\sigma(D(A, V(2))) \geqslant \sigma\left(D_{n}(A, V)\right)-$ $\epsilon=\lambda_{n}(A+V)-\epsilon>0$. Thus $A_{0} \supset A$ implies that $\sigma\left(D\left(A_{0}, V(2)\right)\right)>0$. The definitions of $A_{0}$ and $D\left(A_{0}, V\right)$ make it clear that $x=\left(x_{1}, x_{2}, \ldots\right) \in$ $D\left(A_{0}, V(2)\right)$ if and only if the same is true when any finite number of coordinates of $x$ is changed. Thus, by the 0 -or-1 principle, $\sigma\left(D\left(A_{0}, V(2)\right)\right)=1$ and, by (e), $\lambda_{n}\left(A_{0}+V(3)\right) \geqslant 1-\epsilon$ for $n \geqslant N_{\epsilon}$. Consequently, $\mu\left(A_{0}+V(4)\right) \geqslant 1-\epsilon$ for every $\epsilon>0$ and every symmetric neighborhood $V$ of $0 \in G$. This implies that $\mu\left(A_{0}\right)=1$, and completes the proof.

3. Convergent convolutions. In this section, we consider the analogues of Theorems 2.1 and 2.2 , when $\mu=\mu_{1} * \mu_{2} * \cdots$ is convergent (i.e., (2.6) holds), but not necessarily Cauchy-convergent (i.e., (2.5) need not hold).

For any closed subgroup $H$ of $G$ and $\mu \in P(G)$, define $\mu^{G / H} \in P(G / H)$ by

$$
\mu^{G / H}(A)=\mu\left(T_{H}^{-1} A\right) \text { for any Borel set } A \subset G / H,
$$

where $T_{H}: G \rightarrow G / H$ is the canonical map $g \mapsto H+g$. Then

$$
\left(\mu_{1} * \cdots * \mu_{n}\right)^{G / H}=\mu_{1}^{G / H} * \cdots * \mu_{n}^{G / H}
$$

and 


$$
\nu_{n} \rightarrow \nu \quad \text { in } P(G) \Rightarrow \nu_{n}^{G / H} \rightarrow \nu^{G / H} \quad \text { in } P(G / H) .
$$

The relation (3.3) is clear from the equivalence of (2.1) and (2.2), where $f$ is constant on cosets of $H$ (i.e., $f \in C_{0}^{0}(G / H)$ ).

Proposition 3.1. Let $\mu \in P(G)$ and $H \subset G$ a closed subgroup. (i) If $\mu$ is pure, then $\mu^{G / H}$ is pure and, conversely, if $\mu^{G / H}$ is pure and $H$ is countable, then $\mu$ is pure. (ii) If $\mu$ is purely discontinuous [or absolutely continuous], then $\mu^{G / H}$ is purely discontinuous [or absolutely continuous], and the converse is valid if $H$ is countable. (iii) If $\mu^{G / H}$ is continuous, then $\mu$ is continuous and, conversely, if $\mu$ is continuous and $H$ is countable, then $\mu^{G / H}$ is continuous.

Proof. On (i). If $\mathscr{U}$ is an admissible class of Borel sets on $G$ [or on $G / H$ ], then $T_{H} \mathfrak{A}$ [or $T_{H}^{-1} \mathfrak{Q}$ ] is an admissible class of sets on $G / H$ [or on $G$ ].

Let $\mu$ be pure and let $\mathcal{Q}$ be an admissible class of sets of $G / H$ such that $\mu^{G / H}(A)>0$ for some $A \in \mathfrak{A}$. Then $\mu\left(T_{H}^{-1} A\right)=\mu^{G / H}(A)>0$, so that there is an $A_{0} \in \mathscr{U}$ such that $\mu^{G / H}\left(A_{0}\right)=\mu\left(T_{H}^{-1} A_{0}\right)=1$. Thus, $\mu^{G / H}$ is pure.

Conversely, let $\mu^{G / H}$ be pure and $\mathscr{Q}$ an admissible class of Borel sets of $G$ such that $\mu(A)>0$ for some $A \in \mathscr{A}$. Then $\mu^{G / H}\left(T_{H} A\right) \geqslant \mu(A)>0$ since $T_{H}^{-1}\left(T_{H} A\right) \supset A$. Hence, there is an $A_{0} \in \mathscr{U}$ such that $1=\mu^{G / H}\left(T_{H} A_{0}\right)=$ $\mu\left(T_{H}^{-1}\left(T_{H} A_{0}\right)\right)$. But if $H$ is countable, then $T_{H}^{-1}\left(T_{H} A_{0}\right)$ is the countable union of the sets $A_{0}+h, h \in H$, so that $T_{H}^{-1}\left(T_{H} A_{0}\right) \in \mathcal{A}$. Thus $\mu$ is pure.

On (ii). The statement concerning "purely discontinuous" is clear. If $\mu^{G / H}$ is absolutely continuous and $H$ is countable, then the absolute continuity of $\mu$ follows as in (i).

Let $\mu$ be absolutely continuous and let $\mu^{\prime}(g)$ be its Radon-Nikodým derivative. (The Radon-Nikodým theorem is valid on $G$ even though $\omega_{0 G}$ need not be $\sigma$-finite; cf. [8, (7), p. 256].) Let $C$ be the collection of Borel sets on $G / H$ and $T_{H}^{-1} \mathrm{C}$ the corresponding collection of sets on $G$. Then $\mu^{G / H}$ is absolutely continuous and its Radon-Nikodým derivative is the conditional expectation $E\left(\mu^{\prime} / T_{H}^{-1}\right.$ C); cf. [4, pp. 17-18].

On (iii). This is clear in view of the proof of (i).

Recall that an infinite product $\Pi a_{n}$ of complex numbers is said to be convergent if there is an integer $K$ such that $a_{K} a_{K+1} \cdots a_{n}$ has a nonzero limit as $n \rightarrow \infty$; i.e., if and only if $a_{n} a_{n+1} \cdots a_{N} \rightarrow 1$ as $N \geqslant n \rightarrow \infty$.

Proposition 3.2. If $\mu_{1}, \mu_{2}, \ldots \in P(G)$ and $\Lambda$ is the set of $\gamma \in \Gamma$ for which $\Pi \hat{\mu}_{n}(\gamma)$ converges, then $\Lambda$ is a subgroup of $\Gamma$. If $\mu=\mu_{1} * \mu_{2} * \cdots$ is convergent in $P(G)$, then $\Lambda$ is an open-closed subgroup.

This result is given in Loynes [13, p. 451], who points out that $\Lambda$ is a group in view of the Increments Inequality (2.3) and that $\Lambda$ contains a neighborhood of $0 \in \Gamma$ when $(1.1)$ is convergent since $\hat{\mu}(0)=1 \neq 0$ and $\Pi \hat{\mu}_{n}(\gamma)$ converges 
uniformly on a neighborhood of $\gamma=0$. In the latter case, $\Lambda$ is open-closed; $c f$., e.g., [8, pp. 250-251].

When $\Lambda$ is open-closed, it is the annihilator of its annihilator

$$
H=\{g \in G:(g, \gamma)=1 \text { for all } \gamma \in \Lambda\}
$$

$[15$, p. 36]. Also $H$ is a compact subgroup of $G$ since its dual group $\Gamma / \Lambda$ is discrete; furthermore, the dual group of $G / H$ is $\Lambda[15, \mathrm{pp} .59,35]$.

Theorem 3.1. Let $\mu_{n} \in P(G)$ and $\mu=\mu_{1} * \mu_{2} * \cdots$ be convergent. Then there exists a (unique smallest) compact subgroup $H$ of $G$ such that

$$
\mu^{G / H}=\mu_{1}^{G / H} * \mu_{2}^{G / H} * \cdots
$$

is Cauchy-convergent in $P(G / H)$, i.e.,

$$
\mu_{n}^{G / H} * \cdots * \mu_{N}^{G / H} \rightarrow \omega_{1} \text { as } N \geqslant n \rightarrow \infty .
$$

Furthermore, if $\omega_{0 H}$ is the normalized Haar measure on $H$, considered as a measure in $P(G)$, then

$$
\mu=\mu * \omega_{0 H}
$$

Proof. Let $\Lambda$ be as in Proposition 3.2 and $H$ as in (3.4). The convergence of (3.5) follows from (3.3). Relation (3.6) follows from the definition of $\Lambda$ in Proposition 3.2, from $\hat{\omega} \equiv 1$, and the fact that $\Lambda$ is the dual group of $G / H$. Also, $\gamma \notin \Lambda$ implies that $\hat{\mu}(\gamma)=0$, while $\hat{\omega}_{0 H}(\gamma)$ is 1 or 0 according as $\gamma \in \Lambda$ or $\gamma \notin \Lambda$ $[15$, p. 59]. Thus (3.7) is a consequence of the uniqueness of the FourierStieltjes transform.

THEOREM 3.2. Let $\mu=\mu_{1} * \mu_{2} * \cdots$ be convergent and $H$ a closed subgroup of $G$. (i) If

(3.8) $\prod_{n=1}^{\infty} d_{H n} \neq 0$, where $d_{H n}=\max _{y \in G / H} \mu_{n}^{G / H}(\{y\})=\max _{g \in G} \mu_{n}(g+H)$,

then $\mu^{G / H}$ is not continuous; in which case, $\mu$ is not continuous if $H$ is countable. (ii) Conversely, if $\mu^{G / H}$ is not continuous (e.g., if $\mu$ is not continuous) and $H$ is as in Theorem 3.1, then (3.8) holds. (iii) If $H$ is as in Theorem 3.1 and $\mu^{G / H}$ is not continuous (or, equivalently, (3.8) holds), then $\mu$ is not continuous if and only if $H$ is finite.

Proof. Except for the assertion (iii), this theorem follows from Theorem 2.1, by virtue of Theorem 3.1. The assertion (iii) is a consequence of the following: on the one hand, $\mu$ has an atom if $\mu^{G / H}$ does and $H$ is countable; on the other hand, $\mu=\mu * \omega_{0 H}$ is continuous if $\omega_{0 H}$ is, while $\omega_{0 H}$ is continuous unless $H$ is finite. 
THEOREM 3.3 (PURE THEOREM). Let $\mu=\mu_{1} * \mu_{2} * \cdots$ be convergent in $P(G), \mu_{n}$ purely discontinuous, and $H \subset G$ the compact subgroup of Theorem 3.1. Then $\mu^{G / H}$ is pure (hence absolutely continuous or purely discontinuous or (continuous) singular), and the same is true of $\mu$ if $H$ is finite. Also $\mu=\omega_{0 G}$ if $G$ is compact and $H=G$.

The first part of this theorem follows from Theorem 2.2 by virtue of Theorem 3.1, and the last part from (3.7).

In the important case where $G$ is the circle group $T=R / Z$ and every closed subgroup $H$ is $T$ or is finite, we have

THEOREM 3.4. Let $\mu_{n} \in P(T)$ and $\mu=\mu_{1} * \mu_{2} * \cdots$ be convergent. (i) Then $\mu$ is not continuous if and only if, for some integer $\kappa>0$,

$$
\prod_{n=1}^{\infty} d_{\kappa n} \neq 0, \text { where } d_{\kappa n}=\max _{\theta} \sum_{j=0}^{\kappa-1} \mu_{n}(\{\theta+j / \kappa\}) .
$$

(ii) If, in addition, $\mu_{n}$ is purely discontinuous, then $\mu$ is pure (hence absolutely continuous or purely discontinuous or (continuous) singular); in particular, $\mu$ is purely discontinuous if and only if (3.9) holds for some integer $k>0$.

It is easy to see that (1.2) and (3.9) are not equivalent. For example, in the case that $\omega_{1 / \kappa}=\mu_{1}=\mu_{2}=\cdots$, where $\omega_{1 / \kappa}$ is the probability measure on $T$ with the atoms $0,1 / \kappa, \ldots,(\kappa-1) / \kappa(\bmod 1)$ assigned the equal probability $1 / \kappa$, then $\omega_{1 / \kappa} * \omega_{1 / \kappa}=\mu_{1} * \mu_{2} * \cdots=\omega_{1 / \kappa}$. But $d_{n}=1 / \kappa$ and $d_{\kappa n}=1$.

REMARK. If $\mu_{1}, \mu_{2}, \cdots$ are regular probability measures on $R$ and the result concerning (3.9) is applied to (3.5) with $G=R, H=\epsilon Z$ with $\epsilon>0$, then it follows that $\mu$ is not continuous if and only if

$$
\prod_{n=1}^{\infty} d_{\epsilon n} \neq 0, \text { where } d_{\epsilon n}=\max _{t} \sum_{j=-\infty}^{+\infty} \mu_{n}(\{t+j \epsilon\}),
$$

and so (1.2) and (3.10) are equivalent by Lévy's theorem (when (1.1) is convergent).

4. Additive functions $f: Z_{+} \rightarrow G$. Let $f: Z_{+} \rightarrow G$ be a $G$-valued function on the positive integers $Z_{+}=\{1,2, \ldots\}$. The mean value $M(f)$ is said to exist if $M(f)=\lim N^{-1}[f(1)+\cdots+f(N)]$ exists as $N \rightarrow \infty$. Let $\tau_{N} \in P(G)$ be the distribution of the finite sequence $\{f(1), \ldots, f(N)\}$, i.e.,

$$
\hat{\tau}_{N}(\gamma)=N^{-1}[(f(1), \gamma)+\cdots+(f(N), \gamma)] \text { for } \gamma \in \Gamma .
$$

The function $f$ is said to possess an asymptotic distribution $\mu$ if there exists a $\mu \in P(G)$ and $\tau_{N} \rightarrow \mu$ as $N \rightarrow \infty$ in $P(G)$.

In the remainder of this paper, we suppose that $f: Z_{+} \rightarrow G$ is additive, i.e., $f(m+n)=f(m)+f(n)$ if $m, n$ are relatively prime. 
For fixed primes $p$ and $P$, let $f_{p}(n)$ be the additive function determined by its values on powers of primes given by

$$
f_{p}\left(p^{j}\right)=f\left(p^{j}\right) \text { and } f_{p}\left(q^{j}\right)=0 \text { if } q \neq p \text { is a prime }
$$

and let $f^{P}(n)$ be the additive function

$$
f^{P}(n)=\sum_{p<P} f_{p}(n), \quad \text { so that } f^{P}(n) \rightarrow f(n)=\sum_{p} f_{p}(n) \text { as } P \rightarrow \infty
$$

for $n=1,2, \ldots$. The additive function $f_{p}$ has an asymptotic distribution $\sigma_{p} \in P(G)$, where

$$
\hat{\sigma}_{p}(\gamma)=\left(1-p^{-1}\right)\left[1+\sum_{j=1}^{\infty} p^{-j}\left(f\left(p^{j}\right), \gamma\right)\right],
$$

and $f^{P}$ has the asymptotic distribution $\sigma^{P}=\sigma_{2} * \sigma_{3} * \cdots * \sigma_{P} ; \mathrm{cf}$. [6] for $G=R$.

For fixed $\gamma \in \Gamma$, define the complex-valued multiplicative functions

$$
F_{\gamma}(n)=(f(n), \gamma) \text { and } F_{\gamma}^{P}(n)=\left(f^{P}(n), \gamma\right) \text { for } n=1,2, \ldots
$$

Thus, $f$ has an asymptotic distribution $\mu$ if and only if $M\left(F_{\gamma}\right)$ exists for $\gamma \in \Gamma$ and is continuous at $\gamma=0$ (in which case, $\hat{\mu}(\gamma)=M\left(F_{\gamma}\right)$ ). Note that the convergence of

$$
\sigma_{2} * \sigma_{3} * \cdots * \sigma_{P} * \cdots
$$

to $\mu$ in $P(G)$ is equivalent to $\mu \in P(G)$ and $M\left(F_{\gamma}^{P}\right) \rightarrow \hat{\mu}(\gamma)$, as $P \rightarrow \infty$, for all $\gamma \in \Gamma$.

Halasz's definitive paper [7] concerns the existence of the mean value $M(F)$ of a complex-valued multiplicative function $F,|F(n)| \leqslant 1$. On the one hand, his results and proof (cf. [7, p. 380]) imply that

$$
\sum_{p} p^{-1}\left[1-\operatorname{Re}(f(p), \gamma) p^{-i u}\right]<\infty
$$

holds for at most one real $\mu$. In the case that (4.3) fails for all real $\mu$,

$$
\sum_{p} p^{-1}\left[1-\operatorname{Re}(f(p), \gamma) p^{-i u}\right]=\infty \text { for }-\infty<u<\infty
$$

then $M\left(F_{\gamma}\right)$ exists and is 0 , and also

$$
M\left(F_{\gamma}^{P}\right) \rightarrow 0=M\left(F_{\gamma}\right) \quad \text { as } P \rightarrow \infty \text {. }
$$

On the other hand, a result of Delange (cf. [2] , [3]) and/or of Halasz [7] shows that if

$$
\sum_{p} p^{-1}[1-(f(p), \gamma)] \text { converges, }
$$


then $M\left(F_{\gamma}\right)$ exists and is the convergent product

$$
M\left(F_{\gamma}\right)=\prod_{p}\left\{\left(1-p^{-1}\right)\left[1+\sum_{j=1}^{\infty} p^{-j}\left(f\left(p^{j}\right), \gamma\right)\right]\right\}
$$

so that

$$
M\left(F_{\gamma}^{P}\right)=\prod_{p<P}\{\cdots\} \rightarrow M\left(F_{\gamma}\right) \quad \text { as } P \rightarrow \infty .
$$

As observed by Delange, Halasz's results imply [3, Theorem C, p. 218], which, in turn, has the following consequence.

Proposition 4.1. Let $f: Z_{+} \rightarrow G$ be additive and $\gamma \in \Gamma$ fixed. Then $M\left(F_{\gamma}\right)$ exists and $M\left(F_{\gamma}\right)=0$ if and only if either (4.4) holds or both (4.3) and

$$
2^{-i j u}\left(f\left(2^{j}\right), \gamma\right)=-1 \quad \text { for } j=1,2, \ldots
$$

hold for some real $u$.

A particular case of Delange [2] (see [3, Theorem A, p. 217]) is the following.

Proposition 4.2. Let $f: Z_{+} \rightarrow G$ be additive and $\gamma \in \Gamma$ fixed. Then $M\left(F_{\gamma}\right)$ exists and $M\left(F_{\gamma}\right) \neq 0$ if and only if (4.6) holds and (4.9) fails to hold for $u=0$.

Using arguments of Delange [3], we can obtain the next three propositions.

Proposition 4.3. Let $f: Z_{+} \rightarrow G$ be additive. Let $\Lambda_{0}$ be the set of $\gamma \in \Gamma$ for which there is a (unique) $u=u(\gamma)$ satisfying (4.3). Then $\Lambda_{0}$ is a group and $u(\gamma+\delta)=u(\gamma)+u(\delta)$ for $\gamma, \delta \in \Lambda_{0}$.

Proof. It is convenient to write (4.3) as

$$
\sum_{p} p^{-1} \sin ^{2}[\arg (f(p), \gamma)-u \log p] / 2<\infty .
$$

Thus the assertion follows from $\arg (f(p), \gamma+\delta)=\arg (f(p), \gamma)+\arg (f(p), \gamma)$ and from the simple inequality $\sin ^{2}(x+y) \leqslant 2 \sin ^{2} x+2 \sin ^{2} y$; cf. [3, p. 219].

Proposition 4.4. Let $f: Z_{+} \rightarrow G$ be additive. Let $\Lambda$ be the set of $\gamma \in \Gamma$ satisfying (4.6). Then $\Lambda$ is a subgroup of $\Lambda_{0}$.

Proof. This is contained in Proposition 3.2 since the finite product in (4.8) is $\Pi \hat{\sigma}_{p}(\gamma)$ for $p \leqslant P$. (A direct proof follows by the arguments of Delange [3, pp. 228-229].)

Proposition 4.5. Let $f: Z_{+} \rightarrow G$ be additive and let both $M\left(F_{\gamma}\right)$ and $M\left(F_{\gamma+\gamma}\right)$ exist. Then either (4.4) or (4.6) holds. 
Proof. Suppose that neither (4.4) nor (4.6) holds. Then, by Propositions 4.1 and 4.2, $M\left(F_{\gamma}\right)=0$ and (4.3), (4.9) hold for some $u$. By Proposition 4.3, (4.3) holds if $(\gamma, u)$ is replaced by $(\gamma+\gamma, 2 u)$. But (4.9) does not hold if $(\gamma, u)$ is replaced by $(\gamma+\gamma, 2 u)$. Thus $M\left(F_{\gamma+\gamma}\right) \neq 0$. Consequently, (4.6) is convergent if $\gamma$ is replaced by $\gamma+\gamma$, by Proposition 4.2; so that $\gamma+\gamma \in \Lambda_{0}$ with $u(\gamma+\gamma)=0$, and

$$
\sum_{p} p^{-1} \sin [\arg (f(p), \gamma)] \text { converges }
$$

if $\gamma$ is replaced by $\gamma+\gamma$, i.e., $\arg (f(p), \gamma)$ by $2 \arg (f(p), \gamma)$. Since $\gamma \in \Lambda_{0}$, we have $u(\gamma)=u(\gamma+\gamma) / 2=0$. Thus the real part of (4.6), i.e., (4.10) with $u=0$, is convergent. The imaginary part (4.11) also converges since $|\sin 2 x-2 \sin x|=$ $4|\sin x| \sin ^{2}(x / 2)$. This is contrary to the assumption that (4.6) does not hold, and completes the proof.

For the case $G=R$, Erdös and Wintner [6, (iii), p. 720] show that an additive function $f: Z_{+} \rightarrow R$ has an asymptotic distribution $\mu$ if and only if (4.2) converges. We have the following generalization.

THEOREM 4.1. Let $f: Z_{+} \rightarrow G$ be additive. (i) If $f$ has an asymptotic distribution $\mu$, then (4.2) converges. (ii) Conversely, if (4.2) is Cauchy-convergent, then $f$ has an asymptotic distribution $\mu$, and $\mu$ is pure.

REMARK 1. Theorem 4.3 below for $G=T$ shows that, in general, the convergence of (4.2) is not sufficient for the existence of an asymptotic distribution.

REMARK 2. Since $\sigma_{p}$ is purely discontinuous, the theorems of $\S \S 2-3$ are applicable to (4.2).

Proof. On (i). Since $f$ has an asymptotic distribution $\mu$, it follows that $M\left(F_{\gamma}\right)$ exists and $\hat{\mu}(\gamma)=M\left(F_{\gamma}\right)$ for all $\gamma \in \Gamma$. By Proposition 4.5, we have, for every fixed $\gamma \in \Gamma$, either (4.4), hence (4.5), or (4.6), hence (4.8). Consequently

$$
\lim _{P \rightarrow \infty} \prod_{p<P} \hat{\sigma}_{p}(\gamma)=\hat{\mu}(\gamma) \quad \text { for } \gamma \in \Gamma
$$

This implies the convergence of (4.2).

On (ii). If (4.2) is Cauchy-convergent, then the product in (4.7) is convergent and is $\hat{\mu}(\gamma)$ for all $\gamma$. Hence the series in (4.6) is convergent and (4.7) holds for all $\gamma$, so that $f$ has the asymptotic distribution $\mu$. Also $\mu$ is pure by Theorem 2.2.

THEOREM 4.2. Let $f: Z_{+} \rightarrow G$ be additive and let (4.2) converge. Then the subgroup $\Lambda$ of $\Gamma$ in Proposition 4.4 is the same as the subgroup $\Lambda$ in Proposition 3.2 and Theorem 3.1, where $\mu_{n}=\sigma_{p}$ and $p=p_{n}$; so that if $H$ is the annihilator of $\Lambda$, then $\mu^{G / H}=\sigma_{2}^{G / H} * \sigma_{3}^{G / H} * \cdots$ is Cauchy-convergent. In 
particular, $T_{H} f: Z_{+} \rightarrow G / H$ has an asymptotic distribution $\mu^{G / H}$, and $\mu^{G / H}$ is pure.

This is clear from the proof of Theorem 4.1. For the case of $G=T$, we have the following partial converse to Theorem 4.1(i).

THEOREM 4.3. Let $f: Z_{+} \rightarrow T$ be additive and let (4.2) converge, say, to $\mu$. (i) If $\mu \neq \omega_{0 T}$, then $f$ has the asymptotic distribution $\mu \neq \omega_{0 T}$. (ii) But if $\mu=\omega_{0 T}$, then $f$ need not have an asymptotic distribution.

Remark. Assertion (i) depends on Delange [3, Theorem 2, p. 226], the proof of which implies that (for an arbitrary group $G$ ) if $\Lambda_{0}$ in Proposition 4.3 is $k Z$ and $\Lambda \neq\{0\}$ in Proposition 4.4, then $\Lambda=\Lambda_{0}$. In general, (i) is false if $T$ is replaced by another group $G$, even the torus $G=T \times T$. For let $h: Z_{+} \rightarrow T$ have an asymptotic distribution $\sigma \neq \omega_{0 T}$ and let $f: Z_{+} \rightarrow T$ be as in (ii) above, so that $(h, f): Z_{+} \rightarrow T \times T$ has no asymptotic distribution, but the analogue of (4.2) converges and is $\sigma \times \omega_{0 T} \neq \omega_{0, T \times T}$.

Proof. On (i). The convergence of (4.2) implies that (4.12) holds. If $\mu \neq \omega_{0 T}$, then $\hat{\mu}(\gamma) \neq 0$ for some $\gamma \neq 0$. This implies the convergence of the product in (4.7), hence, of the series in (4.6) for some $\gamma \neq 0$. It follows from [3, Theorem 2, p. 226] that $f$ has an asymptotic distribution. This distribution is (4.2) by Theorem 4.1.

On (ii). It will be shown that if $h: Z_{+} \rightarrow R$ is additive,

$$
h(p)=\log p \quad \text { and } h\left(p^{j}\right)=0 \text { for } j>1,
$$

and $f: Z_{+} \rightarrow T$, where $f(n)=h(n) \bmod 1$, then (4.2) is convergent with $\mu=$ $\omega_{0 T}$, but $f$ does not have an asymptotic distribution. In order to see this, note that if $F_{m}(n)=\exp 2 \pi i m f(n)=\exp 2 \pi i m h(n)$, then

$$
\begin{aligned}
M\left(F_{m}^{P}\right) & =\prod_{p \leqslant P}\left(1-p^{-1}\right)\left[1+p^{-1} e^{2 \pi i m \log p}\right] \\
& =\exp \left\{-\sum_{p \leqslant P} p^{-1}\left(1-e^{2 \pi i m \log p}\right)+O(1)\right\}
\end{aligned}
$$

as $P \rightarrow \infty$. Hence

$$
\left|M\left(F_{m}^{P}\right)\right|=\exp \left\{-2 \sum_{p \leqslant P} p^{-1} \sin ^{2} \pi m \log p+O(1)\right\}
$$

As shown by Delange $[3$, p. 221$]$,

$$
\sum_{p} p^{-1} \sin ^{2} \pi m \log p=\infty \text { for } m=1,2, \ldots,
$$

so that $M\left(F_{m}^{P}\right) \rightarrow 0$ as $P \rightarrow \infty$ for $m=1,2, \ldots$ This gives (4.2) with $\mu=\omega_{0 T}$. 
Thus, by Theorem 4.1, it follows that if $f$ has an asymptotic distribution, then this distribution is $\omega_{0 T}$. But then Propositions 4.1 and 4.5 imply that (4.4) holds for all $\boldsymbol{\gamma}$, i.e.,

$$
\sum_{p} p^{-1}\left[1-\operatorname{Re} F_{m}(p) p^{-i u}\right]=\infty
$$

$$
\text { for }-\infty<u<\infty \text { and } m=1,2, \ldots \text {, }
$$

[3, Theorem 1, p. 220]. However, if $u=2 \pi m$, then $F_{m}(p) p^{-i u}=1$, by (4.16), which contradicts (4.14). This completes the proof.

Theorem 3.4 will be seen to have the following consequences.

THEOREM 4.4. Let $f: Z_{+} \rightarrow R$ be a real, additive function such that $f$ $\bmod 1: Z_{+} \rightarrow T$ has an asymptotic distribution $\mu$. Then (i) $\mu$ is pure (hence purely discontinuous or absolutely continuous or singular), and (ii) $\mu$ is purely discontinuous if and only if there exists an integer $\kappa>0$ such that

$$
\sum_{\{p: k f(p) \neq 0 \bmod 1\}} p^{-1}<\infty .
$$

Part (ii) contains the corrected version of the theorem in [5] giving a necessary and sufficient condition for $\mu$ to be continuous.

A prime $p$ does not occur in the sum (4.15) if the number $f(p)$ is of the form $f(p)=($ integer $)+j / \kappa$ for $j=0,1, \ldots$, or $k-1$. When $\mu \neq \omega_{0 T}$, then $\mu$ is purely discontinuous if and only if (4.15) holds, where $\mu$ is chosen so that $0,1 / \kappa, \ldots,(\kappa-1) / \kappa(\bmod 1)$ is the subgroup $H$ of Theorem $4.2 ;$ cf. [3, pp. 227-229], where $\kappa=q$.

The necessary condition (4.15) cannot be replaced by

$$
\sum_{\{p: f(p) \neq 0 \bmod 1\}} p^{-1}<\infty .
$$

In order to see this, it suffices to exhibit a real additive function $f$ possessing an asymptotic distribution mod 1 satisfying (4.15) for some $\kappa>1$ (so that $\mu$ is purely discontinuous), but not satisfying (4.16). To this end, let $\kappa>1$ be fixed and let $f$ be a real additive function defined by $f(p)=1 / k$ and $f\left(p^{j}\right)=0$ for $j>1$ for every prime $p$. Then the analogue of (4.6),

$$
\sum_{p} p^{-1}[1-\exp 2 \pi i m f(p)] \text { converges }
$$

for every $m$ divisible by $\kappa$, so that $f$ has an asymptotic distribution mod 1 [ 3 , Theorem 2, p. 226]. Note that $f(p) \neq 0 \bmod 1$ for every $p$, so that (4.16) fails. But (4.15) holds since the sum is over an empty set of primes.

Proof of Theorem 4.4. On (i). Part (i) is a consequence of Theorem 3.3 
for $\sigma_{p}$ is purely discontinuous, since the $m$ th Fourier-Stieltjes coefficient of $\sigma_{p}$ is (1.3) with $u=2 \pi m$.

On (ii). By (1.3), the jump $\sigma_{p}(\{0\})$ is at least $1-p^{-1} \geqslant 1 / 2$. Thus the maximum jump $d_{k p}$ occurs at $\theta=0$ and is

$$
\left(1-p^{-1}\right)\left[1+\epsilon_{\kappa p} p^{-1}+O\left(p^{-2}\right)\right]=1+\left(\epsilon_{\kappa p}-1\right) p^{-1}+O\left(p^{-2}\right),
$$

as $p \rightarrow \infty$, where $\epsilon_{\kappa p}$ is 1 or 0 according as $\kappa f(p)$ is or is not $0 \bmod 1$. Hence (4.15) is equivalent to $\Pi d_{\kappa p} \neq 0$, and so part (ii) follows from Theorem 3.4.

\section{REFERENCES}

1. H. Cartan and R. Godement, Théorie de la dualité et analyse harmonique dans les groupes abéliens localement compacts, Ann. Sci. École Norm. Sup. (3) 64 (1947), 79-99. MR 9, 326.

2. H. Delange, Sur les fonctions arithmétiques multiplicatives, Ann. Sci. École Norm. Sup. (3) 78 (1961), 273-304. MR 30 \#72.

3. - On the distribution modulo 1 of additive functions, J. Indian Math. Soc. 34 (1970), 215-235.

4. J. L. Doob, Stochastic processes, Wiley, New York; Chapman \& Hall, London, 1953. MR 15, 445.

5. P. T. D. A. Elliott, The continuity of the limiting distribution of additive functions $(\bmod 1)($ to appear).

6. P. Erdös and A. Wintner, Additive arithmetical functions and statistical independence, Amer. J. Math. 61 (1939), 713-721. MR 1, 40.

7. G. Halász, Über die Mittelwerte multiplikativer zahlentheoretischer Funktionen, Acta Math. Acad. Sci. Hungar. 19 (1968), 365-403. MR 37 \#6254.

8. P. R. Halmos, Measure theory, Van Nostrand, Princeton, N. J., 1950. MR 11, 504.

9. B. Jessen and A. Wintner, Distribution functions and the Riemann zeta function, Trans. Amer. Math. Soc. 38 (1935), 48-88.

10. E. R. van Kampen, Infinite product measures and infinite convolutions, Amer. J. Math. 62 (1940), 417-448. MR 1, 209.

11. P. Lévy, Sur les séries dont les termes sont des variables éventuelles indépendantes, Studia Math. 3 (1931), 119-155.

12. M. Loève, Probability theory. Foundations. Random sequences, 3rd ed., Van Nostrand, Princeton, N. J., 1963. MR 34 \#3596.

13. R. M. Loynes, Products of independent random elements in a topological group, Z. Wahrscheinlichkeitstheorie und Verw. Gebiete 1 (1962/63), 446-455. MR 27 \#6293.

14. V. A. Rohlin, On the fundamental ideas of measure theory, Mat. Sb. 25 (67) (1949), 107-150; English transl., Amer. Math. Soc. Transl. (1) 10 (1962), 1-54. MR 11, 18.

15. W. Rudin, Fourier analysis on groups, Interscience Tracts in Pure and Appl. Math., no. 12, Interscience, New York, 1962. MR 27 \#2808.

16. A. Wintner, Asymptotic distributions and infinite convolutions, Lecture Notes, The Institute for Advanced Study, Princeton, N. J., 1938.

17. The Fourier transforms of probability distributions, Lecture Notes, Baltimore, 1947.

DEPARTMENT OF MATHEMATICS, THE JOHNS HOPKINS UNIVERSITY, BALTIMORE, MARYLAND 21218 